\title{
Complication of epiduroscopy: a brief review and case report
}

\author{
${ }^{1}$ Unit of Pain Therapy, Salvatore Maugeri Foundation, Scientific Institute of Pavia, Pavia, \\ ${ }^{2}$ Anaesthesia, Intensive Care and Pain Therapy Service, Azienda Ospedaliera Universitaria Parma Hospital, Parma, \\ ${ }^{3}$ Department of Surgical Sciences, Azienda Ospedaliera Universitaria Parma Hospital, University of Parma, Parma, Italy
} Maurizio Marchesini ${ }^{1}$, Edoardo Flaviano ${ }^{2}$, Valentina Bellini ${ }^{2}$, Marco Baciarello ${ }^{2}$, and Elena Giovanna Bignami ${ }^{3}$

Epiduroscopy is defined as a percutaneous, minimally invasive endoscopic investigation of the epidural space. Periduroscopy is currently used mainly as a diagnostic tool to directly visualize epidural adhesions in patients with failed back surgery syndrome (FBSS), and as a therapeutic action in patients with low back pain by accurately administering drugs, releasing inflammation, washing the epidural space, and mechanically releasing the scars displayed. Considering epiduroscopy a minimally invasive technique should not lead to underestimating its potential complications. The purpose of this review is to summarize and explain the mechanisms of the side effects strictly related to the technique itself, leaving aside complications considered typical for any kind of extradural procedure (e.g. adverse reactions due to the administration of drugs or bleeding) and not fitting the usual concept of epiduroscopy for which the data on its real usefulness are still lacking. The most frequent complications and side effects of epiduroscopy can be summarized as non-persistent post-procedural low back and/or leg discomfort/pain, transient neurological symptoms (headache, hearing impairment, paresthesia), dural puncture with or without post dural puncture headache (PDPH), post-procedural visual impairment with retinal hemorrhage, encephalopathy resulting in rhabdomyolysis due to a dural tear, intradural cyst, as well as neurogenic bladder and seizures. We also report for first time, to our knowledge, a case of symptomatic pneumocephalus after epiduroscopy, and try to explain the reason for this event and the precautions to avoid this complication. (Korean J Pain 2018; 31: 296-304)

Key Words: Cicatrix; Epidural space; Failed back surgery syndrome; Low back pain; Neurogenic urinary bladder; Paresthesia; Pharmaceutical preparations; Pneumocephalus; Post-dural puncture headache; Retinal hemorrhage; Tissue adhesions.

\section{INTRODUCTION}

Epiduroscopy is a minimally invasive technique, useful for assessing the epidural space through a flexible instrument connected to an optical fiber. The possibility of exploring the epidural space with direct vision allows both the clinical evaluation of scars in the spinal canal and intervertebral foramina (as in the failed back surgery syndrome - FBSS) and in so-called "virgin back" patients with channel stenosis; in addition to simple clinical evaluation, the epiduroscopic treatment allows targeted delivery of drugs, debridement of adhesions, and reduction of inflammatory

Received August 16, 2018. Revised September 13, 2018. Accepted September 13, 2018.

Correspondence to: Maurizio Marchesini

Unit of Pain Therapy, Salvatore Maurgeri Foundation, 4 street Salvatore Maugeri, Scientific Institute of Pavia 27100, Italy

Tel: +39-03825921, Fax: +39-0382592504, E-mail: marchesinidoc@gmail.com

(a) This is an open-access article distributed under the terms of the Creative Commons Attribution Non-Commercial License (http:// creativecommons.org/licenses/by-nc/4.0/), which permits unrestricted non-commercial use, distribution, and reproduction in any medium, provided the original work is properly cited.

Copyright (C) The Korean Pain Society, 2018 
factors with abundant lavage.

The first report on an endoscopic study of the anatomy of the spinal canal dates back to Burman [1] in the early 1930s. He used arthroscopic equipment and vertebral columns removed from cadavers. A few years later, American neurosurgeon Pool reported on over 400 spinal endoscopies. No further reports of similar techniques are found in the literature until 1967. These early studies specifically inspected the intrathecal space.

In time, the technique came to be primarily used for examining the epidural space, thanks to the development of small-caliber flexible optics by the end of the 1980s. Shimoji et al. [2] added two important features to the technique: performing epiduroscopy under conscious sedation and identifying the affected nerve root by touching it and reproducing the patient's pain. In 1994, Saberski and Kitahata described the caudal approach, which greatly reduced the risk of dural puncture. They also were the first to describe the use of a flexible, steerable epiduroscope, and irrigation of the epidural space with saline to aid visualization. In 1996, the US Food and Drug Administration approved epiduroscopy for visualizing the epidural space.

Epiduroscopy, as a diagnostic tool, plays a unique role for its "functional" nature: the reproducibility of the pain for which the patient is seeking treatment (concordant pain) through manipulation of specific epidural structures informs us that a given region of the spinal canal is directly or indirectly related to the symptoms of interest. As compared to clinical evaluation and MRI, epiduroscopy has shown greater specificity indicating the actual vertebral level of clinically-significant spinal pathology. In addition, epiduroscopy has been shown to be more sensitive than MRI in detecting epidural fibrosis. According to Bosscher and Heavner [3] of the 78 patients with persistent pain after spinal surgery who underwent epiduroscopy, preoperative MRI showed epidural fibrosis in $16.1 \%$ the $\mathrm{pa}^{-}$ tients, whereas epiduroscopy demonstrated fibrosis to various extents in $91 \%$ of them. If scarring was reported to be present on MRI, severe fibrosis was found on epiduroscopy as well. The reverse was not true. These finding suggest that MRI may not be a sensitive tool in the diagnosis of epidural fibrosis.

As for "targeted treatments", direct lysis of adhesions and scar tissue represents one the prevailing application, utilizing both pharmacological and physical means. Adhe- siolysis is typically performed in the context of failed back surgery syndrome, which remains the most common indication for epiduroscopy.

Considering epiduroscopy a minimally invasive technique should not lead to underestimating its potential complications. The purpose of this review is to summarize and explain the mechanisms of the side effects strictly related to the technique itself, leaving aside complications considered typical for any kind of extradural procedure (e.g. adverse reactions due to the administration of drugs or bleeding) and not fitting the usual concept of epiduroscopy for which the data on its real usefulness are still lacking. (e.g. adjuvant treatments like laser, radiofrequency, and Fogarty's catheter).

\section{CASE REPORT}

We report for first time, as far as we know, a case of symptomatic pneumocephalus after epiduroscopy. Written informed consent was obtained from the patient for publication of this case report and any accompanying images.

The patient was a 52-year-old male undergoing epiduroscopy with a diagnosis of FBSS. He had undergone a posterior lumbar interbody fusion (PLIF) for L4-L5 spondylolisthesis 2 years prior. The indication for the intervention was given in relation to the symptoms of the $\mathrm{pa}^{-}$ tient who reported severe spinal neurogenic claudication of about 100 meters and intense lumbar pain (NRS $=8-10$ ) in an upright position and during postural changes. Surgery was complicated by an epidural hematoma and residual cauda equina syndrome with pelvic paresthesias and a neurological bladder. These symptoms were being treated at another center.

The patient then reported a worse symptomatology after the operation, both for the onset of new neurological symptoms and for the persistence of spinal claudication and lumbar and radicular neuropathic pain, substantially unchanged.

Magnetic resonance imaging 1 year after PLIF showed satisfactory union of the vertebrae and correct alignment of the fusion hardware including the interbody cage, as well as the L4 and L5 pedicle screws and rods. Inflammatory tissue surrounding the dural sac reached laterally to occupy the L4-L5 foramina on both sides, and anteriorly, coming into contact with the residual intervertebral disc. The cauda equina was displaced posteriorly at the L4-L5 
level, a finding consistent with post-surgical adhesive arachnoiditis (Fig. 1).

In February 2017, an epiduroscopy was performed in an attempt to clear adhesions between the dura and vertebrae, which should have reduced traction on the dural sac. With the patient in the prone position, standard monitoring was applied and an area around the sacral hiatus was prepped for epidural access. Monitored anesthesia care included propofol and remifentanil infusions, titrated to $\mathrm{pa}-$ tient comfort while maintaining verbal contact.

The epiduroscopy was performed with the Resascope system (AMSgroup srl, Padua, Italy) by an expert pain physician who had performed over 80 procedures at the time.

The Resascope set is composed of a $10 \mathrm{~F}$ adjustable epidural video-guide catheter with an external diameter of $3.3 \mathrm{~mm}$, a length of $30 \mathrm{~cm}$, and 2 channels, one for saline infusion for washing the epidural space, thus improving visibility, and a second one for collection of the fluid, three entry channels for insertion of flexible fiberoptic and other tool, an internal diameter of $1.25 \mathrm{~mm}$ (Fig. 2), as well as an introducer and service kit with different syringes, a 17G Touhy needle (for sacral epidural access), a guide wire for the Seldinger technique, a $12 \mathrm{~F}$ rigid dilatator and $10 \mathrm{~F}$ introducer, and a gallipot of $250 \mathrm{ml}$ for saline aspiration (Fig. 3). The kit includes a bag allowing the option of more accurately measure the outgoing volume. The procedure lasted $70 \mathrm{~m}$, with a final saline infusion volume of $350 \mathrm{ml}$.

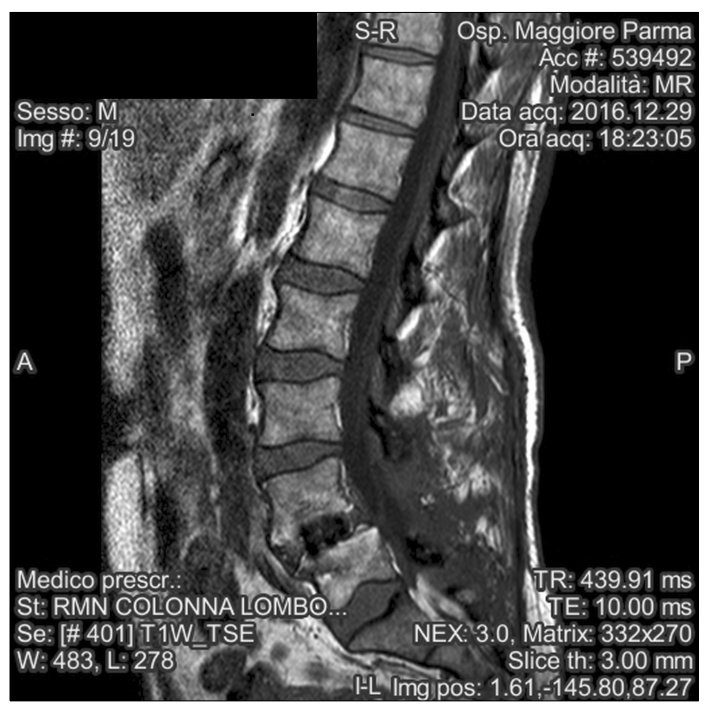

Fig. 1. MRI lumbar scan before periduroscopy.
During the procedure several areas of fibrosis (mostly grade II and III per the Bosher and Heavner classification) were reported at the L4-L5 interspace; grade II fibrosis with remarkable hyperemia was found at the L3-L4 level. Debridement from adhesions of the spinal canal was performed at each level using a balloon catheter with the maximum diameter reached by the inflated balloon being $5 \mathrm{~mm}$, whereas the use of radiofrequency resection was avoided because of the history of epidural bleeding. The epidural space was explored to the level of Ll; no signs of a dural tear were noted. At the end of the procedure, the propofol/remifentanil sedation was stopped and the patient, in a prone position, was calm, in complete contact, and did not report any unusual sensation

A few seconds after he was returned to the supine position and his head raised about $30^{\circ}$, the patient complained of intense paresthesia in his lower extremities, accompanied by muscle rigidity. One minute later he became confused, unresponsive, with arterial blood pressure rising

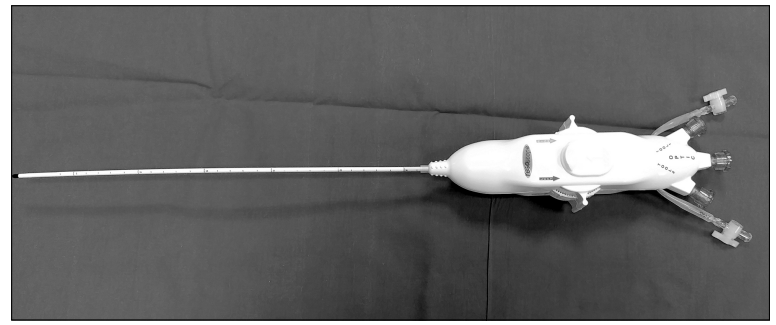

Fig. 2. Flexible Epidural Videoguide.

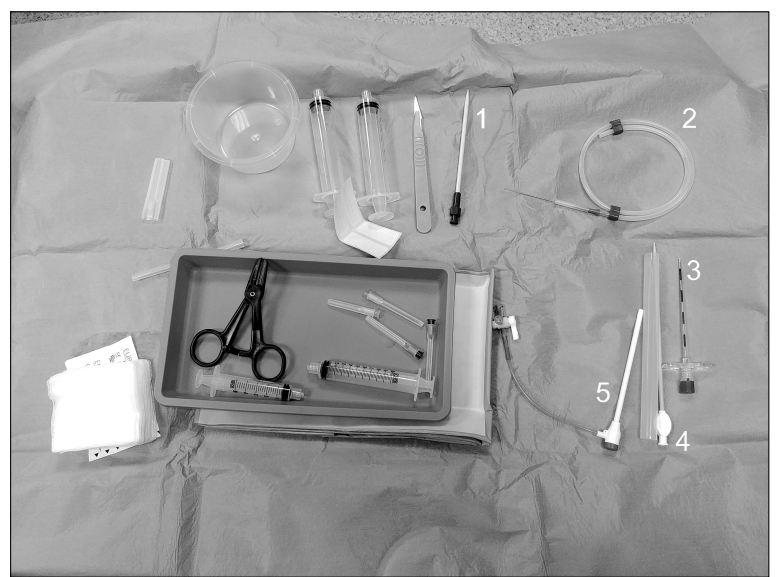

Fig. 3. Introducer Kit: 1: Rigid dilatator 8 F; 2: guide wire 0,38 ' $\times$ 50; 3: Touhy needle 17G; 4: rigid dilatator 12 F; 5: introducer $10 \mathrm{~F}$. 
over $180 / 100 \mathrm{mmHg}$.

Bag-mask ventilation with 100\% oxygen was initiated but found to be unnecessary, as the airway was patent, with acceptable respiratory activity. Urapidil and clonidine were carefully titrated to control the blood pressure. Betamethasone $8 \mathrm{mg}$ was administered because of the initial suspicion of painful radicular irritation. Minutes later, an intravenous infusion of propofol was started to keep the patient calm due to the agitation that the sensation of intense paresthesias, especially in the limbs, and the marked tachycardia, had caused him.

Despite the treatments, 30 minutes after the initial event, our patient still exhibited an altered mental status. A brain CT scan was ordered, and the patient was more deeply sedated and intubated to ensure immobility during the exam.

The scan was positive for air bubbles homogeneously distributed among the subarachnoid cisterns of the skull base; a previously-unknown cystic malformation in the posterior fossa was also reported (Fig. 4, 5).

The patient was transferred to the surgical intensive care unit. Since there was no indication of increased intracranial pressure or parenchymal damage, the patient was warmed and gradually weaned from sedation and mechanical ventilation. He was extubated about 3 hours after the initial incident but still 1 hour after he was in contact, in pressure support ventilation with normalized hemodynamic values.

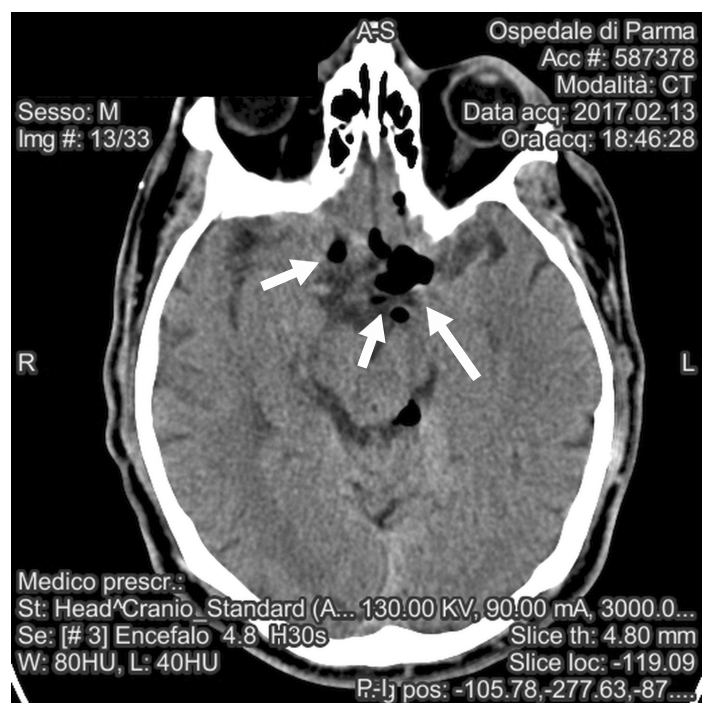

Fig. 4. CT brain scan, white arrows show airbubbles.
Later on the same day, the patient was transferred to the surgical ward and finally discharged 2 days later, free of symptoms. He was seen at follow-up visits 1, 3, and 6 months after the procedure, where he reported a global improvement (reduction in back pain and in spinal claudication) of $80 \%, 50 \%$ and $50 \%$, respectively. His Oswestry disability index score improved by similar amounts.

The PubMed, Scopus, and ISI Web of Science databases were systematically searched to find articles related to epiduroscopy. Since there is lack of uniformity in literature among the correct definition of the maneuver, common and unusual synonyms of the procedure were also sought 'periduroscopy, epiduraloscopy, extraduroscopy, and spinal endoscopy'. The last computer search was performed in April 2018. No language restrictions were included but only English articles were reviewed. The research produced limited results and, considering the paucity of studies with the previous mentioned key words included in the title and/or in the abstract, all articles were examined for reports of complications in the results or discussion sections. The reference sections of prime articles were then searched to obtain additional references.

A total of 87 studies were retrieved, dating from 1985 to 2018, using the previously mentioned search strategy. Reviews and studies on animals and cadavers were excluded, and the remaining articles were examined independently by 2 authors (M.M. and E.F.) to find any

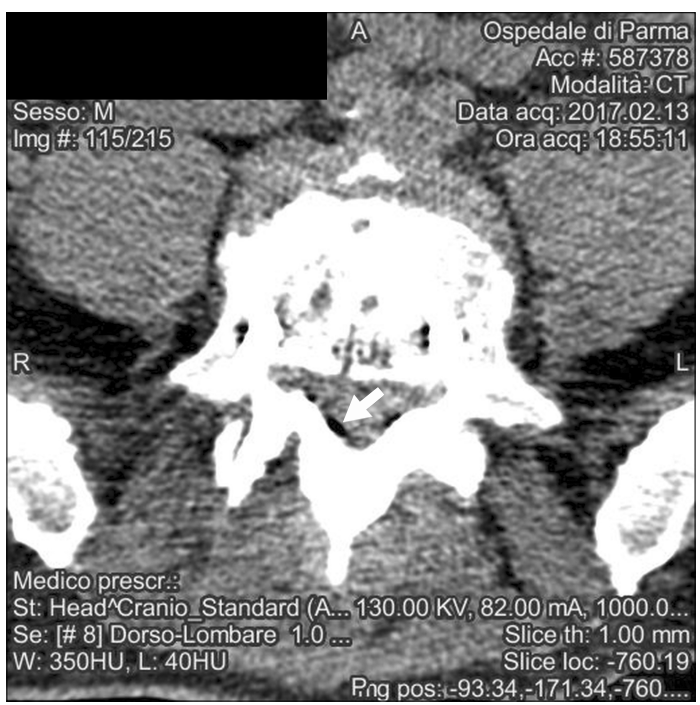

Fig. 5. CT scan of L4-L5 level, white arrow shows airbubbles. 
documented complications to the epiduroscopic technique. At the end, a total number of 14 articles were selected (Fig. 6). The selected articles have been schematically gathered in a table (Table 1) [4-17] following a chronological order.

These articles include 6 prospective studies (176 patients), 1 retrospective study (60 patients and 77 procedures), and 8 case reports. In total, epiduroscopy was performed on 244 patients of whom 135 (55\%) had FBSS and 109 (45\%) had not undergone previous back surgery.

Complications and side effects ranged from minimal and often self-limited transient neurologic symptoms to severe manifestations, sometimes long lasting, for which further diagnostic workups and specific care were necessary (e.g. encephalopathy).

According with data of this review, complications and side effects of epiduroscopy can be summarized, with respect to their own prevalence, as follow:

1. Non-persistent post-procedural low back and/or leg discomfort/pain similar to that encountered with other percutaneous techniques performed at the same level. In the vast majority of cases, the symptomatology lasted for a few days and showed a good response to simple oral an- algesics; the clinical conditions never being sufficient to require admission to the hospital.

2. Transient neurological symptoms (headache, hearing impairment, paresthesia) generally related to the increase in intracranial pressure due to the administration of a certain amount of saline solution at a specific rate. All symptoms regressed in a few seconds after the immediate interruption of saline infusion, but paresthesia sometimes lasted for few days.

3. Dural puncture (13 cases) with post dural puncture headace (PDPH) (2 cases).

4. Suspected infections (8 cases), never confirmed, for which an empiric therapy was administered.

5. Post-procedural visual impairment with retinal hemorrhages and intravascular injection (2 cases each).

6. Encephalopathy resulting in rhabdomyolysis due to a dural tear (1 case), intradural cyst (1 case), neurogenic bladder (lcase) and seizures (1 case);

7. Technical inability to perform the procedure in $3 \mathrm{pa}^{-}$ tients, who were given caudal epidural injections instead.
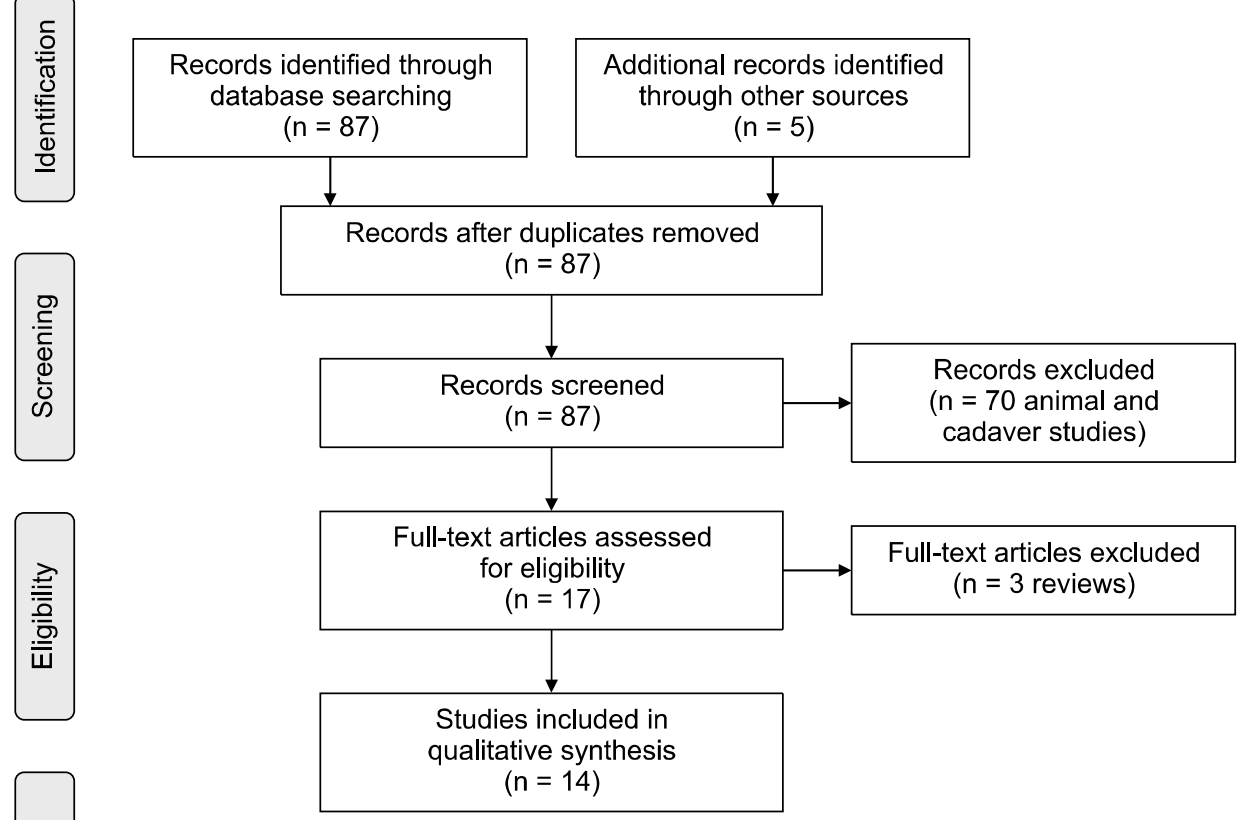
Table 1. Studies Included in the Review

\begin{tabular}{|c|c|c|}
\hline Author & Article & Complications \\
\hline $\begin{array}{l}\text { Avellanal and Diaz-Reganon } \\
\text { (2008) [4] }\end{array}$ & $\begin{array}{l}\text { Prospective study ( } \mathrm{n}=19 \text { patients with severe } \\
\text { FBSS) }\end{array}$ & $\begin{array}{l}\text { Dural puncture in } 4 \text { patients (only } 1 \text { PDPH). } \\
\text { TNS (headache and transient hypoacousia) in } 4 \\
\text { patients. } \\
\text { Pos-tprocedural low back and leg discomfort in } \\
\text { some patients }\end{array}$ \\
\hline Manchikanti et al. (1999) [5] & $\begin{array}{l}\text { Retrospective study ( } n=60 \text { patients with FBSS } \\
\text { and } 77 \text { epiduroscopic procedures) }\end{array}$ & $\begin{array}{l}\text { Suspected infection in } 8 \text { cases } \\
\text { Dural puncture in } 7 \text { cases }\end{array}$ \\
\hline Amirikia et al. (2000) [6] & Case report & Acute bilateral visual loss with retinal hemorrhages \\
\hline Richardson et al. (2001) [7] & Prospective study ( $\mathrm{n}=38$ patients, 19 with FBSS) & $\begin{array}{l}\text { Transient Neurological Symptoms in } 2 \\
\text { patientsNon-persistent post procedural low back } \\
\text { discomfort in all patients }\end{array}$ \\
\hline Igarashi et al. (2004) [8] & $\begin{array}{l}\text { Prospective study ( } n=58 \text { patients with } \\
\text { degenerative lumbar spinal stenosis without } \\
\text { previous back surgery) }\end{array}$ & Dural puncture in 1 patient (without PDPH) \\
\hline Dash field et al. (2005) [9] & $\begin{array}{l}\text { Prospective study ( } \mathrm{n}=30 \text { patients with chronic } \\
\text { sciatica without previous back surgery) }\end{array}$ & $\begin{array}{l}\text { Impossible caudal approach in } 3 \text { cases } \\
\text { Non-persistent postprocedural low back discomfort } \\
\text { in all patients }\end{array}$ \\
\hline Mizuno et al. (2007) [10] & Case report (FBSS) & $\begin{array}{l}\text { Dural tear, encephalopathy, and rhabdomyolysis } \\
\text { induced by contrast media iotrolan injected into } \\
\text { the CSF }\end{array}$ \\
\hline Heavner et al. (2007) [11] & Case report (2 cases with FBSS) & Intravascular injection \\
\hline Moschos et al. (2008) [12] & Case report & $\begin{array}{l}\text { Postprocedural bilateral visual loss with retinal and } \\
\text { vitreous hemorrhages }\end{array}$ \\
\hline Justiz et al. (2010) [13] & Case report (FBSS) & Neurogenic bladder \\
\hline Ryu et al. (2012) [14] & Case report (FBSS) & Intradural cyst \\
\hline Magalhães et al. (2013) [15] & Prospective study ( $n=13$ patients with FBSS) & $\begin{array}{l}\text { Headache in } 1 \text { patient }<24 \mathrm{~h} \\
\text { Paresthesia in lower limbs during ozone application }\end{array}$ \\
\hline Avellanal et al. (2014) [16] & $\begin{array}{l}\text { Prospective study ( } n=18 \text { patients with severe } \\
\text { FBSS) }\end{array}$ & $\mathrm{PDPH}$ in 1 patient \\
\hline Beyaz (2015) [17] & Case report & Seizures and TNS \\
\hline
\end{tabular}

\section{DISCUSSION}

Reports of complications related to epiduroscopy are sparse and limited, typically described as minor and self-limited; however, serious events are still possible. In this review 20 out 244 patients (8\%) presented with one of these major complications (dural puncture, visual impairment, intravascular injection, intradural cyst, and seizures). All but one (a case of neurogenic bladder) were reversible, with no mortality.

The most frequent manifestations include transient neurological symptoms (TNSs) and post-procedural pain in the affected area mostly related to nerve irritation and tissue inflammation, similar to those shown in conventional percutaneous adhesiolysis and other procedures performed in the spinal canal.

Physiologically normal intracranial pressure is at the level of 5-10 mm $\mathrm{Hg}$ at rest. Following extradural injection of $10 \mathrm{ml}$, intracranial pressure increased to between 11 and $63 \mathrm{mmHg}[16,17]$. This increase in pressure occurs within seconds of injection, and may continue to increase pressure for approximately 45 seconds. Increased intracranial pressure has been shown to return to normal levels between 2 minutes, 20 seconds and 5 minutes, 50 seconds [17]. This increase in pressure may be greater in patients with a priori above-average intracranial pressure and in general with poor compliance (this is often the case in patients with FBSS or degenerative spinal pathology), in which a $10 \mathrm{ml}$ epidural injection can cause a more serious increase in pressure, up to approximately $300 \mathrm{mmHg}$. 
The upper limit of the amount of saline which can be safely injected during epiduroscopy is not known. One hundred cc is often used, although a range from $60 \mathrm{~mL}$ to $250 \mathrm{ml}$ has been proposed. Therefore, the amounts of steroid, hyaluronic acid, contrast medium, and saline administered during epiduroscopy should be calculated precisely, and the presence of headache should be assessed intermittently, as it is reported to be an indicator of increased intracranial pressure in patients under monitored anesthesia care [17].

The increase in the epidural hydrostatic pressure and, consequently, in the intracranial pressure, due both to the total amount of fluid injected (saline, drugs) and the infusion rate, is the common pathogenic mechanism at the origin of transient neurological symptoms. (including headache, neck pain and hypoacusia). They typically subside seconds after the discontinuation of the epidural infusion, however, if this doesn't occur within 5 minutes [3] or if TNSs recur with low-pressure flushes [15], the procedure should be discontinued. Communication between patient and physician is extremely important, especially in these phase, hence the need for monitored anesthesia care. Gill and Heavner [18] reported that epidural lavage should be administered at a low speed of $1 \mathrm{~mL} / \mathrm{s}$ : a volume of 100 $\mathrm{ml} / 60 \mathrm{~min}$ should not be exceeded, and an infusion rate of $0.03 \mathrm{ml} / \mathrm{s}$ is appropriate.

With similar mechanism visual disturbance may develop as a consequence of retinal hemorrhages, mainly of venous origin, caused by epidural injection of fluid and the subsequent increase in intracranial pressure (ICP). Two mechanisms have been proposed: firstly, an increase in cerebral spinal fluid (CSF) pressure may be transmitted directly through the dural sheaths around the optic nerve into the retinal venous circulation. Secondly, the decrease in blood flow due to ICP increase may activate a reflex increase in ophthalmic artery pressure with resultant venous collapse and the rupture of capillaries [6]. Complete recovery of vision after venous retinal hemorrhage usually occurs over a matter of days to months. Permanent loss of acuity may occur after massive hemorrhages or if atrophy develops as a consequence of bleeding into the optic nerve. There is no treatment for retinal venous hemorrhages unless they involve the vitreous, in which case vitrectomy may eventually be indicated $[18,19]$. In the two cases reporting visual impairment, visual acuity was improved, although it was found to still be limited 4-6 months after epiduroscopy.

In a recent review on complications of epiduroscopy, Avellanal and Diaz-Reganon [4] proposed "the 60 limit rule": procedures lasting no more than 60 minutes, while not exceeding $60 \mathrm{ml}$ of injected volume, and $60 \mathrm{mmHg}$ of epidural pressure. This approach could be suitable for preventing both TNSs (including seizure, considered the most severe form of TNSs) and visual impairment, by acting on their common mechanism.

According to this review, the incidence of dural puncture is higher in patients with FBSS (93\%) as opposed to patients affected by degenerative pathology (mostly spinal canal stenosis) and no history of back surgery. The differences between the two groups may be due to more challenging anatomy in patients with FBSS, but it should be noted that a potential bias exists in the study by Avellanal and Diaz-Reganon [4], whereby a different technique (interlaminar epidural access) was employed in patients with FBSS. Equipment was also different: a $0.77 \mathrm{~mm} \mathrm{flex-}$ ible fiberscope, $150 \mathrm{~cm}$ in length, covered with a $4 \mathrm{~F}$ angiographic catheter (outer diameter 1.35, inner diameter 0.97 $\mathrm{mm}$ ), which was connected to a Y-adapter/hemostasis valve for fluid administration, was introduced via the epidural needle. The final report was 4 dural punctures: two happened during catheter advance and the other two upon needle entry. The author considers the former two a side effect during the lysis of adhesions, but the latter two could have been affected by the choice of epidural approach.

Surprisingly just two cases of post-dural puncture headache (PDPH) out of 13 dural punctures were registered, much less than expected considering the diameter of the instrument, the level of the puncture (lumbar), and the low compliance of the epidural space. A reasonable explanation could be that adhesions, scars, and injected saline (leaking through the breach into the subarachnoid space) may act to minimize CSF loss [4].

In one case it was reported that after a dural iatrogenic lesion, an intradural cyst developed of as a consequence of a ball valve phenomenon of CSF leakage. Mizuno et al. [10] reported an episode of rhabdomyolysis linked to a contrast medium injection. Also, in this case the passage of the contrast medium into the subarachnoid space was caused by invisible breaches, and the patient's neurological symptoms were very similar to those of the clinical case presented here. All these conditions strongly 


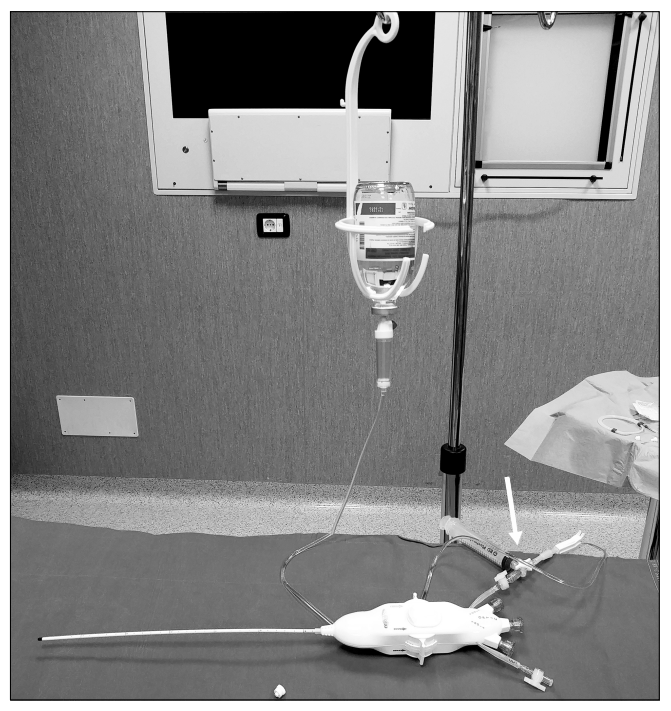

Fig. 7. Fitted close circuit for fluid injection.

remind us of the importance of considering the possibility of an accidental dural tear in cases of the appearance of both new neurological symptoms and an inexplicable increase in the intensity of pain, refractory to medical management. It is important to consider such complications even if the usual maneuvers to detect a dural tear (direct visualization with an epiduroscope, aspiration of CSF from the epidural space, and contrast medium injection under fluoroscopy) are negative.

Encephalopathy and neurologic bladder were strictly related to the administration of, respectively, contrast medium and hypertonic saline/local anesthetic. According to Avellanal and Diaz-Reganon [4], any unexpected neurologic sign or symptom must be kept under strict clinical monitoring until complete recovery is seen. Urgent MRI or CT scans should be obtained when in doubt.

As for infections, these are rare complications with an incidence comparable to other epidural and spinal procedures, nevertheless some physicians prefer to administer an antibiotic as prophylaxis; this is a personal preference, not supported by evidence.

To the best of our knowledge, this is the first published case of pneumocephalus as a consequence of endoscopic epidurolysis. We hypothesize that the complication happened because of minimal interruptions of dural integrity which were invisible through the small fiber optic endoscope used for epiduroscopy; such lesions could be micro-tears from previous stabilization surgery, or new ones caused by the physical lysis of tenacious adhesions.
Despite this complication, the clinical result was good, with pain relief and an improvement in quality of life comparable to that of other published works, including the recent retrospective study by Hazer et al. [20] with similar rates and timing.

Air entry into the epidural space was conceivably in the form of microbubbles in the injectate and/or of leaks in the suction tubing. We postulate that air microbubbles were clinically silent as long as our patient was kept in a horizontal position (either prone or supine). Once his head and torso were elevated in the bed, bubbles floated rostrally and coalesced due to reduced available space and hydrostatic pressure.

Pneumocephalus may be one of the rarest complication of epiduroscopy described to date; yet, our case demonstrates that it is something to take into account when $\mathrm{pa}^{-}$ tients present with altered mental status after the procedure. We also performed a review of our equipment and the way we use it for epiduroscopy, which resulted in switching to a closed suctioning system which we now purge from air before starting the procedure (Fig. 7).

\section{REFERENCES}

1. Burman MS. Myeloscopy or the direct visualization of the spinal canal and its contents. J Bone Joint Surg 1931; 13: 695-6.

2. Shimoji K, Fujioka H, Onodera M, Hokari T, Fukuda S, Fujiwara $\mathrm{N}$, et al. Observation of spinal canal and cisternae with the newly developed small-diameter, flexible fiberscopes. Anesthesiology 1991; 75: 341-4.

3. Bosscher HA, Heavner JE. Incidence and severity of epidural fibrosis after back surgery: an endoscopic study. Pain Pract 2010; 10: 18-24.

4. Avellanal M, Diaz-Reganon G. Interlaminar approach for epiduroscopy in patients with failed back surgery syndrome. $\mathrm{Br} J$ Anaesth 2008; 101: 244-9.

5. Manchikanti L, Pampati V, Bakhit CE, Pakanati RR. Nonendoscopic and endoscopic adhesiolysis in post-lumbar laminectomy syndrome: a one-year outcome study and cost effectiveness analysis. Pain Physician 1999; 2: 52-8.

6. Amirikia A, Scott IU, Murray TG, Halperin LS. Acute bilateral visual loss associated with retinal hemorrhages following epiduroscopy. Arch Ophthalmol 2000; 118: 287-9.

7. Richardson J, McGurgan P, Cheema S, Prasad R, Gupta S. Spinal endoscopy in chronic low back pain with radiculopathy. A prospective case series. Anaesthesia 2001; 56: 454-60.

8. Igarashi T, Hirabayashi Y, Seo N, Saitoh K, Fukuda H, Suzuki 
H. Lysis of adhesions and epidural injection of steroid/local anaesthetic during epiduroscopy potentially alleviate low back and leg pain in elderly patients with lumbar spinal stenosis. Br J Anaesth 2004; 93: 181-7.

9. Dashfield AK, Taylor MB, Cleaver JS, Farrow D. Comparison of caudal steroid epidural with targeted steroid placement during spinal endoscopy for chronic sciatica: a prospective, randomized, double-blind trial. $\mathrm{Br} J$ Anaesth 2005; 94: 514-9.

10. Mizuno J, Gauss T, Suzuki M, Hayashida M, Arita $H$, Hanaoka K. Encephalopathy and rhabdomyolysis induced by iotrolan during epiduroscopy. Can J Anaesth 2007; 54: 49-53.

11. Heavner JE, Wyatt DE, Bosscher HA. Lumbosacral epiduroscopy complicated by intravascular injection. Anesthesiology 2007; 107: 347-50.

12. Moschos MM, Rouvas A, Papaspirou A, Apostolopoulos M. Acute visual loss and intraocular hemorrhages associated with endoscopic spinal surgery. Clin Ophthalmol 2008; 2: 937-9.

13. Justiz R, Taylor $V$, Day M. Neurogenic bladder: a complication after endoscopic adhesiolysis with return of bladder function while using nitrofurantoin. Anesth Analg 2010; 110: 1496-8.
14. Ryu KS, Rathi NK, Kim G, Park CK. latrogenic intradural lumbosacral cyst following epiduroscopy. J Korean Neurosurg Soc 2012; 52: 491-4.

15. Magalhães FN, Soares SC, Torres JM, Ungaretti A, Cacciacarro MF, Teixeira MJ, et al. Effects of ozone applied by spinal endoscopy in patients with chronic pain related to failed back surgery syndrome: a pilot study. Neuropsychiatr Dis Treat 2013; 9: 1759-66.

16. Avellanal M, Diaz-Reganon G, Orts A, Soto S. One-year results of an algorithmic approach to managing failed back surgery syndrome. Pain Res Manag 2014; 19: 313-6.

17. Beyaz SG. Seizures and transient neurological deficits during epiduroscopy in a patient with failed back surgery syndrome. Pain Med 2015; 16: 825-7.

18. Gill JB, Heavner JE. Visual impairment following epidural fluid injections and epiduroscopy: a review. Pain Med 2005; 6: 367-74.

19. Bolder PM, Norton ML. Retinal hemorrhage following anesthesia. Anesthesiology 1984; 61: 595-7.

20. Hazer DB, Acarbaş A, Rosberg HE. The outcome of epiduroscopy treatment in patients with chronic low back pain and radicular pain, operated or non-operated for lumbar disc herniation: a retrospective study in 88 patients. Korean J Pain 2018; 31: 109-15. 